\title{
PARAMETERIZATION OF LASER BURNED SAMPLES AND ITS USAGE IN DATA DESCRIPTION AND SIMULATION
}

\author{
Jana Hájková \\ Department of Computer Science and Engineering \\ University of West Bohemia \\ Univerzitní 8, Pilsen, Czech Republic \\ E-mail: hajkovaj@kiv.zcu.cz
}

\section{KEYWORDS}

Parameterization, laser burning, model, simulation, approximation, surface.

\begin{abstract}
This paper describes the reasons and methods for parameterization of samples burned by a laser beam. At the beginning, it gives information about data acquisition and description. In the main part, the paper summarizes the basic set of parameters which describe the shape of a pulse. From these parameters the basic shape is generated according to a mathematical description. The method of sample approximation is described in detail. Finally, the future plans are outlined.
\end{abstract}

\section{INTRODUCTION}

Our work is a part of a larger project that deals with laser burning, its control, and simulation of the process of laser burning. Simulation of the burning process should prevent real burning of pointless experiments. As an input into the simulation model real burned and measured data sets are used, but samples have to be processed first. We are interested in data preprocessing and its visualization. For our work, we use real samples burned into several materials and measured by a confocal microscope. The way of data acquisition is described in the following section.

All measured samples are represented in the form of a height map. Its format is described later in section Data Description. Our task is to visualize the samples and to design algorithms for automatic preprocessing and comparison of samples which will be later used for simulation purposes. Because the height map is not an optimal format for all these operations that we need to perform, we try to describe the sample in a different way. To recognize differences and similarities among similar samples, we had to explore the real samples carefully. The results of this process are described in the first part of the Results section. Our goal is to find a set of parameters that would define a mathematical function approximating the shape of the pulse optimally and to get a parametrical description of sample roughness in all its areas.

If we are able to describe the real sample by a set of parameters, we can use the parameterization as a part of the sample generating process. First, we generate the basic shape of the pulse. To get more realistic results, the smooth surface generated by the function can be further modified by usage of random surface modification. For that we plan to use methods of waves modulation as well as methods of noise generation. Because the main idea of this paper is the exploration of real samples, their parameterization and the generation of the basic pulse shape, these methods are only outlined in the section Future Plans.

After a careful exploration of real samples, we can try to find the dependence of their parameters on the number of laser pulses burned during the real sample creation. Finally, we should be able to compute parameters for any arbitrary sample and to generate its surface in the process of simulation. In other words, we should be able to simulate the appearance of any sample based only on a limited set of samples. The surface of a 3D function can be used for the generation of any pulse described by the set of parameters as a method of the sample simulation.

\section{Data Acquisition}

To get data for the simulation input, real samples have to be burned by an existing laser equipment into a real material and then to be measured. The set of the samples is called an experiment. One experiment contains data for one particular laser device and one particular material. It means that for each combination of a laser device and a material which are used for simulation, a special experiment has to be performed. Each experiment consists of samples burned by the laser into a single point in the material. The number of pulses goes in sequence, e.g. from 1 to 100 (in Figure 1, each pulse from the sequence is burned on a separate row).

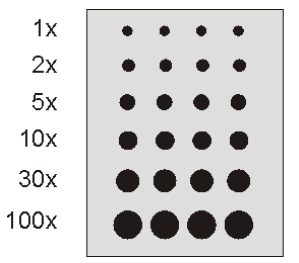

Figure 1: Experiment Layout

After reburning the same pulse several times with keeping the same conditions, the results differ a little, so each pulse count is repeated several times in order to get 
an average result. These samples are placed side by side in a single row (one row in Figure 1 corresponds to similar pulses). All data we use is burned by a laser device BLS-100 (Nd:YAG solid-material, lamp-pumped laser with wavelength of $1064 \mathrm{~nm}$ ) into steel and cermet (a composite material composed of ceramic and metallic materials). Parameters of burning are as follows: laser power $100 \mathrm{~W}$, current $28 \mathrm{~A}$, width of the ray $0.01 \mathrm{~mm}$, diaphragm 1.8.

After the samples are burned, they have to be measured. For this purpose, the confocal microscope Olympus LEXT OLS3100 is used. During the measurement, just a part of the material with burned pulses is focused, scale and zoom factors are chosen, the material sample is scanned and the measured data is saved. For the output from the confocal microscope, text file (CSV) is used. The zoom is chosen according to the experience with the particular material.

\section{Data Description}

The CSV file contains a matrix of real numbers which represents the sample surface. The values express heights of intersection points in the uniform rectangular grid. This grid represents a height map which describes the surface of the sample (see Figure 2).

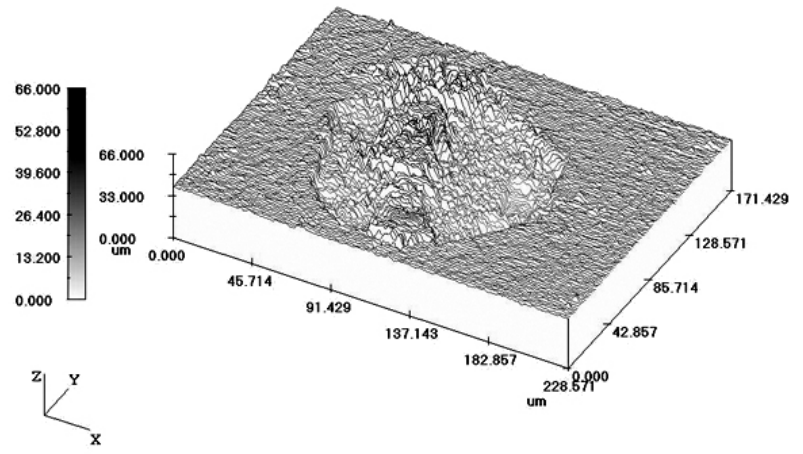

Figure 2: Height Map of a Measured Sample

The majority of samples which we use has the same size as the sample in Figure 2. Most of the sample surface is filled by the area that was affected by the laser beam; the real dimension of the sample is $256 \times 192 \mu \mathrm{m}$. The grid of height map is really fine; most common grid step in used data is $0.25 \mu \mathrm{m}$. It means that the surface of such sample is described by $1024 \times 768$ values.

Let's explore the process of laser burning and material ablation in detail. During the burning process, the surface of the material is exposed to an intense pulsed laser beam that creates a rapid rise in local temperature. The surface is warming up and the material starts to ablate. The material, which is ablated, redeposites around the irradiated area and damages the surrounding material. Finally, at the exposure site, a pit with a transition ring around it is left behind (Dahotre et al. 2008). The cross-section of sample can be seen in Figure 3.

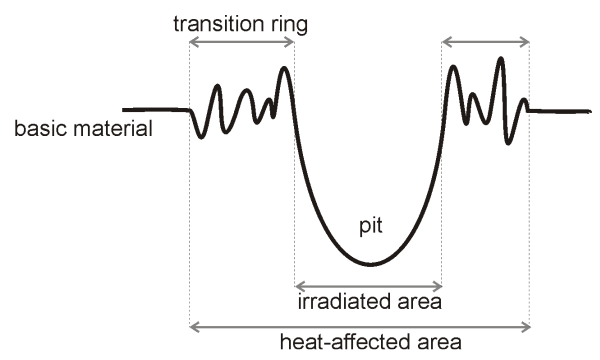

Figure 3: Cross-Section of a Sample and its Description

As can be seen in Figure 4, the real samples can be visualized in various ways. Different approaches enable various opportunities and we can see different properties there. The confocal microscope itself enables the 3D visualization (see Figure 2). In Figure 4, two different approaches of the sample visualization by our tool are shown. The grayscale image in Figure $4 \mathrm{a}$ is used for the sample exploration combined with the vertical and horizontal cross-sections of the sample surface. It gives a good imagination about distribution of heights and depths in the surface. Figure $4 \mathrm{~b}$ shows the result of our $3 \mathrm{D}$ viewer. It enables to explore the surface from an arbitrary position of the camera. Thanks to the plasticity and shades, the output is more realistic than the previous approach. The top view of the sample is shown in Figure 4b. In both these images, the same sample (100 laser pulses burned into a steel surface) is shown. a)

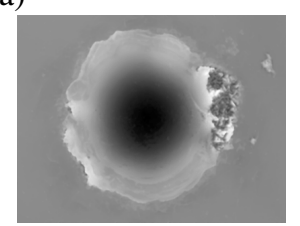

b)

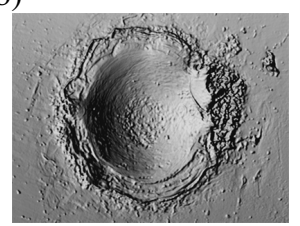

Figure 4: Visualization of a Sample: a) as a Grayscale Image, b) as a 3D View

Let's describe the sample surface in detail. The surface of the original material is quite smooth with some local roughness (Figure 5a), the centre of the pulse (i.e. the area irradiated by the laser beam) is also smooth and the bottom of the pit is a little bit rougher (Figure $5 \mathrm{~b}$ ). The most ragged surface part is the transition region. The surface is modulated by some concentric waves that are both regular and irregular (Figure 5c). Sometimes, local defects with a considerable roughness can appear, especially at the outer border of the transition ring (Figure 5d). They are caused by the ablated material that becomes cool and deposits in the area next to the pit irregularly. At the outer border, the roughness declines slowly and it goes into the roughness of the bulk material.

All these facts should be taken into account if we want to generate the surface of a pulse from its parameters. Samples burned into the same material are similar and some characteristics of the burned sample depend directly on the used material itself. If we burn the same 
sample twice, it will be never the same because at least the surface of the basic material differs. That is why it is not necessary to save the exact height map, but it is sufficient to save characteristics and attributes of the sample. It is the main reason for the parameterization of samples. a)

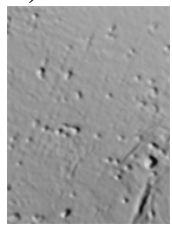

c)

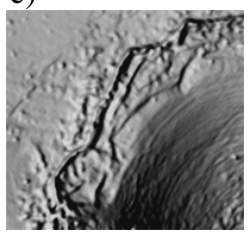

b)

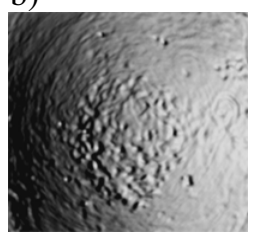

d)

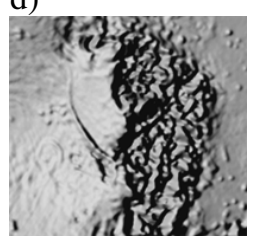

Figure 5: Typical Examples from Different Parts of a Typical Sample Surface

The set of sample parameters is described in the following section. If we want to generate the sample surface from these parameters, we have to, at the beginning, get the basic shape of the heat affected area. This procedure is described as the main idea of this paper in the section Pulse Surface Generating.

\section{PULSE PARAMETERIZATION}

To get information about the pulse shape, we use crosssection lines. Figure 6 shows the cross-section curves of four similar samples of steel with 100 pulses burned into one place. Because pulses are not symmetric, we cannot use cross-sections measured only in one direction. Our experiments show that cross-sections measured in two directions - in the broadest and the narrowest width of the pulse, are sufficient. Thanks to the character of pulses the cross-sections are often orthogonal and correspond to cross-section parallel to $x$ and $y$ axis. For simplification let's call them $\mathrm{CS}_{\mathrm{X}}$ and $\mathrm{CS}_{\mathrm{Y}}$. a)

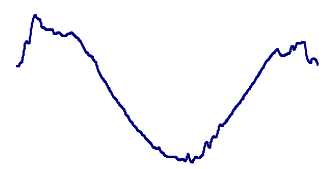

c)

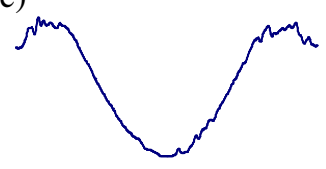

b)

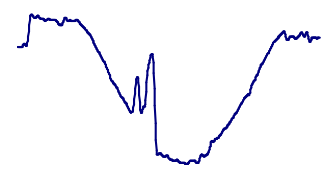

d)

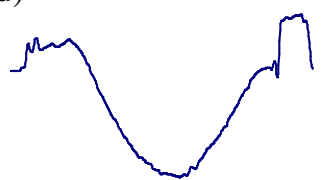

Figure 6: Vertical Cross-Section Lines of Four Similar Samples - 100 Laser Pulses Burned into the Steel
It is evident that all samples are most similar in the area of the pit. In Figure $6 b$, the shape of the pit is damaged by a local defect of the material, but still the correct shape can be guessed well. The surface of the pulse in the pit is quite smooth and the slight roughness is perceptible in the bottom part. On the contrary, the border of the pulse - the transition ring, which is formed by the melted material, is different for each explored case.

The aim of parameterization is to get a set of parameters that describes the sample sufficiently. Some of them depend on the material and so they do not have to be gained automatically (such parameters are marked with an asterisk in Table I), the others differ for each sample. The parameters can be grouped according to the area. The names of parameters used in the following description are given in parenthesis behind the description.

Table I: Parameters for the Sample Representation

\begin{tabular}{|l|l|}
\hline \multirow{3}{*}{$\begin{array}{l}\text { basic } \\
\text { material }\end{array}$} & basic material level (materialLevel) \\
\cline { 2 - 2 } pit & diameter, height and density of bumps * \\
\hline \multirow{3}{*}{$\begin{array}{l}\text { transition } \\
\text { ring }\end{array}$} & $\begin{array}{l}\text { diameter (semimajor and semiminor axis } \\
\text { of the ellipse) }(a, b)\end{array}$ \\
\cline { 2 - 2 } & ablation depth (pitDepth) \\
\cline { 2 - 2 } & roughness of the pit bottom * \\
\cline { 2 - 2 } & the half of the ring width (ringRadius) \\
\cline { 2 - 2 } & $\begin{array}{l}\text { average height (ringHeight) } \\
\text { height, width (will be not solved in this } \\
\text { paper yet) }\end{array}$ \\
\cline { 2 - 2 } & parameters for local defects * \\
\hline
\end{tabular}

In the simplified cross-section line of the sample we can detect several important points. Points $A\left[x_{A}, y_{A}\right]$ and $B\left[x_{B}, y_{B}\right]$ state the outer border of the pulse pit, $A^{\prime}\left[x_{A}\right.$, $\left.y_{A},\right]$ and $B^{\prime}\left[x_{B}, y_{B},\right]$ determine the outer border of the transition ring. Point $S\left[x_{S}, y_{S}\right]$ gives position of the bottom of the pit. All points are shown in Figure 7. The symbols will also be used in the following computations.

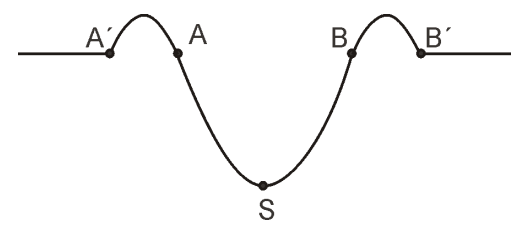

Figure 7: Important Points in the Cross-Section Line

For the detection of these points algorithms for the pulse automatic detection described in (Hájková 2008) are also used. If the area of the pulse is detected, the crosssection lines from the broadest part of the pulse in both the vertical and the horizontal directions are used. From them, the points are derived. 
The level of basic material can be determined according to methods described in (Hájková 2008). The inner diameter of the ring corresponds to the diameter of the pit and the width of the transition ring is computed from the cross-section lines as in the case of the pit. The half of the ring width is called the ring diameter.

More problematic task is to compute the maximal height of the ring, because the surface of real samples is in this area very rough, so we cannot use only the maximal value of the ring cross-section. But if we discretize the quadratic function, we can compute the average value of the parabola. As can be seen in Figure 8, where the average value is shown as the dashed line, the result depends on the sampling frequency. a)

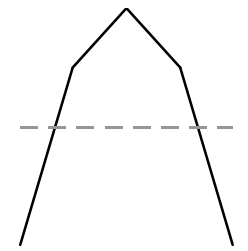

b)

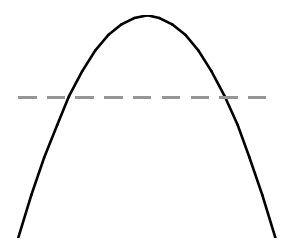

Figure 8: The Position of the Average Value in the Parabola Represented by a) 5 values; b) 21 values.

Because we need to compute the average height of the transition ring, we have to use the same frequency that was used previously for the cross-section of the pit. First, we compute the rate of the average value and the difference of the maximal and the minimal value (amplitude) in the discretized parabola and we get the relative position of the average value from the top of the parabola. This value is independent from the height multiplicator $a$ of the parabola in the parabola definition $\left(f(x)=-a x^{2}\right)$. That is why we can use the expression (1) to compute the amplitude (the height) of the transition ring of the pulse.

$$
\frac{\text { functionAverage }}{\text { functionAmplitude }}=\frac{\text { ringAverage }}{\text { ringHeight }}
$$

\section{PULSE SURFACE GENERATING}

Because both parts of the pulse (i.e. the pit and the ring) differ quite a lot, we have decided to approximate them separately by two different functions and most especially to differentiate the way of surface roughness description. Both generated surfaces are finally connected into the final shape. In following sections the method is derived (Rektorys 1981), (Weisstein 1999).

\section{Pit Approximation}

After several experiments, it appears that the basic shape of the pit cross-section corresponds to the shape of the plot of a quadratic function. The cross-section can be interlaid by a parabola having the equation showed in the expression (2). The results of this parabolic approximation of the pulse pit can be seen in Figure 9.

$$
y=\frac{y_{A}-y_{S}}{\left(x_{A}-x_{S}\right)^{2}} \cdot\left(x-x_{S}\right)^{2}+y_{S}
$$

a)

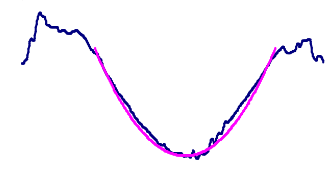

c)

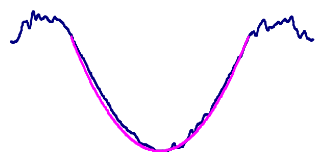

b)

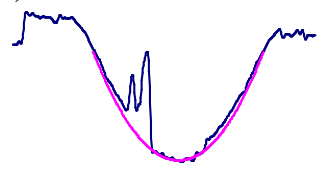

d)

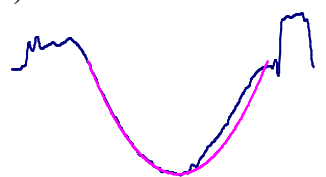

Figure 9: Vertical Cross-Section Lines of Samples from Figure 6 and their Approximation by a Parabola

From the given points measured in both $\mathrm{CS}_{\mathrm{X}}$ and $\mathrm{CS}_{\mathrm{Y}}$, we can compute the other characteristics of the pit, such as its depth (pitDepth) or diameters of the pit in the broadest $(a)$ and the narrowest $(b)$ place. As can be seen in Figure 10, in the real samples of 10, 50 and 100 laser pulses burned into steel, the pit has a circular or elliptical shape from the top view. a)

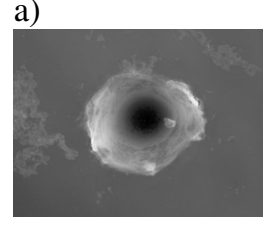

b)

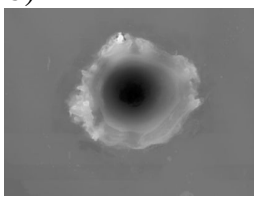

c)

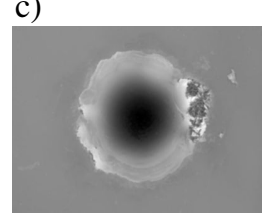

Figure 10: Samples of 10, 50 and 100 Laser Pulses Burned into the Steel from the Top View

If the pulse pit can be approximated by the parabola in its cross-section, the whole pit can be approximated by an elliptical paraboloid. Because we need the paraboloid going through the top border of the pit, the equation representing it has to be modified as in (3). The origin of the solid lies in $S\left[x_{0}, y_{0}, z_{0}\right]$, pitDepth represents the depth of the paraboloid and $a, b$ are axis of the ellipse. The elliptical paraboloid is depicted in Figure 11.

$$
z=\text { pitDepth } *\left(\frac{\left(x-x_{0}\right)^{2}}{a^{2}}+\frac{\left(y-y_{0}\right)^{2}}{b^{2}}\right)+z_{0}
$$

\section{Ring Approximation}

The approximation of the area of pulse transition ring is much more problematic, because its shape is irregular and rough (as can be seen from samples in Figure 6). The material surface is created during the burning process by the ablated material that forms a ring on the border of the burned pit as the material is getting colder. To find the approximating function, we had to simplify it and then to find a suitable way of random noise generation to get as realistic results as possible. 


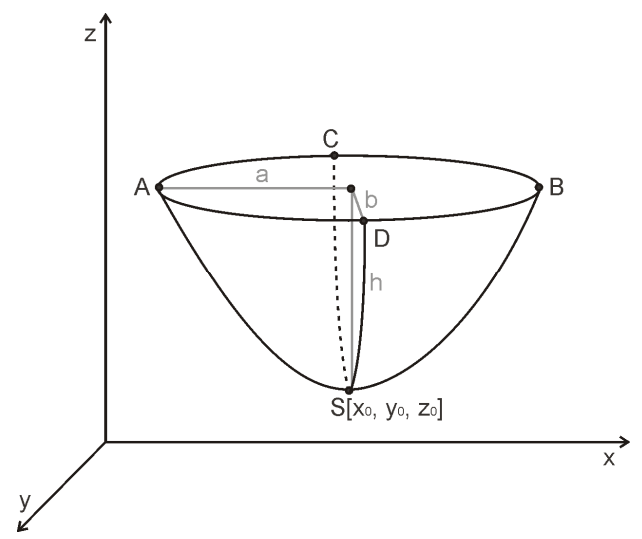

Figure 11: Elliptical Paraboloid and its Parameters.

First, we had to find a suitable function that could be used for the approximation of the basic shape of the ring cross-section. We have decided once again to use the quadratic function. To find an appripriate parabola, we need several parameters to describe the ring. These are shown in Figure 12 - the ring radius and its maximal height and also the level of the basic material. If we have computed all these parameters, we can define the parabola according to the expression (4).

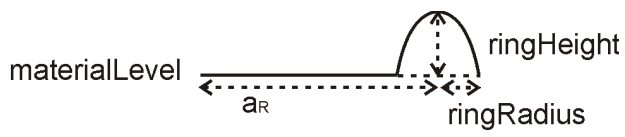

Figure 12: Important Parameters for the Description of the Ring Cross-Section

$$
y=\frac{\text { ringHeight }}{\text { ringRadius }^{2}} *\left(x-a_{R}\right)^{2}+\text { ringHeight }+ \text { materialLevel }
$$

The whole ring should be approximated by a 3D function. It seems to be roughly similar to the top half of a torus. A torus is a surface of revolution generated by revolving a circle in three dimensional space about an axis coplanar with the circle, which does not touch the circle. A torus is shown in Figure 13. Of course, for our purposes, it has to be modified.

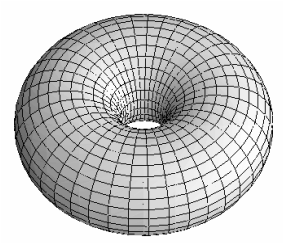

Figure 13: A Torus.

Because the ring has an elliptical shape from the top view and the shape of a parabola from the cross-section, the solid representing the ring should be created as a surface of revolution generated by revolving a parabola along the elliptic trajectory in three dimensional space.
The approximation of the whole ring can be done by the half of the parabolic elliptic torus. The ellipse and its axes are shown in Figure 14.

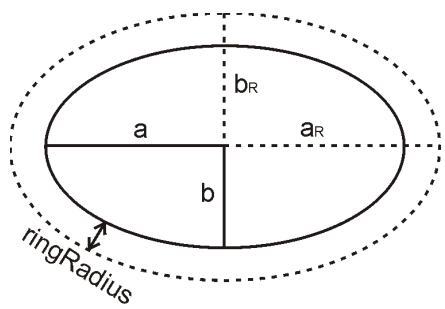

Figure 14: Description of the Ellipse Axis

Now we have to describe it mathematically. The ellipse is defined as (5), where $u$ and $v$ are the axis of the ellipse.

$$
1=\frac{\left(x-x_{0}\right)^{2}}{u^{2}}+\frac{\left(y-y_{0}\right)^{2}}{v^{2}}
$$

The maximum of the parabola representing the ring has to be placed on this ellipse. We can define $u^{2}=a_{R}$ and $v^{2}=b_{R}$. For our case, we can use the equation (6).

$$
p=\frac{\left(x-x_{0}\right)^{2}}{a_{R}}+\frac{\left(y-y_{0}\right)^{2}}{b_{R}}
$$

The value $p=0$ represents the center of the ellipse, $p=$ 1 defines the ellipse itself. If $p$ varies from 0 to 1 , it represents the area bordered by the ellipse and all values $p>1$ points outside the ellipse.

Next, we need an expression for distance of a point from another point. Morespecifically, we need to compute the distance of the processed point from the center $\left[\mathrm{x}_{0}, \mathrm{y}_{0}\right]$ during the surface generation. This distance has to be adjusted according to the ellipse and so for the next computation the value $d_{T}$ will be used. The value computed according to the expression (7) determines the distance of the parabola maximum from the center of the ellipse in a given direction (as can be seen in Figure 15).

$$
d_{T}=\frac{\sqrt{\left(x-x_{0}\right)^{2}+\left(y-y_{0}\right)^{2}}}{p}
$$

a)

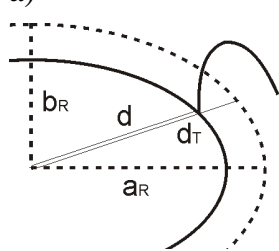

b)

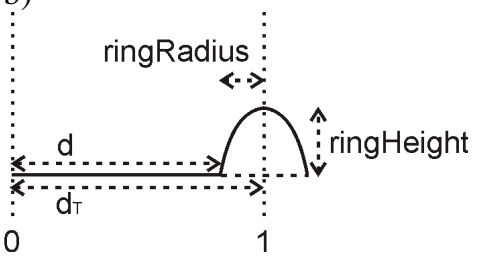

Figure 15: Description of Distances: a) from the Top View, b) from the View of Ring Cross-Section

To get the right shape of the parabola, we have to compute the parameter $k$ that will determine the opening of the parabola (8). It depends on the maximal ring 
height and its radius that has to be recalculated by $d_{T}$ to get the ratio to the ellipse distance.

$$
k=\frac{\text { ringHeight }}{\left(\frac{\text { ringRadius }}{d_{T}}\right)^{2}}
$$

Now we can substitute into the expression (4). Because we compute in recounted distance (in the interval $<0,1>$ ) instead of the value $a_{R}$, we have to use the value 1. Finally, the torus is defined as (9). The constants ringHeight and materialLevel are added because of shifting the generated surface into the right height. Otherwise, it would be placed under the zero level.

$$
z=-k *(p-1)^{2}+\text { ringHeight }+ \text { materialLe vel }
$$

\section{RESULTS}

In the first section of the results section, the parameter comparison of the real samples is made. In the second section, the results of the pulse approximation are shown, both in the form of pulse cross-section (Figure 17 ) and as the 3D view (Figure 18).

\section{Pulse Parameterization}

As a part of the parameterization process, we have to explore the real samples carefully. As written in the section Data Acquisition, the same number of laser pulses burned into the same material several times under the same conditions does not give the same results and that is why each pulse count burning is repeated several times. First, we need to know how much the similar samples differ. To show concrete numbers, the basic parameters computed for five similar samples with 100 laser pulses burned into the steel were summarized in Table II. Moreover, the average values for each single parameter are also computed.

Table II: Basic Parameters of Five Similar Samples $[\mu \mathrm{m}]$

\begin{tabular}{|l|l|l|l|l|l|}
\hline & $a$ & $b$ & $\begin{array}{l}\text { pit } \\
\text { depth }\end{array}$ & $\begin{array}{l}\text { ring } \\
\text { width }\end{array}$ & $\begin{array}{l}\text { ring } \\
\text { height }\end{array}$ \\
\hline$A$ & 39.0 & 43.13 & 8.44 & 13.66 & 2.68 \\
\hline$B$ & 38.37 & 44.25 & 9.89 & 14.5 & 1.99 \\
\hline$C$ & 39.63 & 44.38 & 8.94 & 12.63 & 1.93 \\
\hline$D$ & 38.13 & 46.38 & 8.5 & 12.35 & 1.51 \\
\hline$E$ & 43.88 & 46.0 & 9.39 & 12.63 & 3.89 \\
\hline average & 39.8 & 44.83 & 9.0 & 13.15 & 2.4 \\
\hline
\end{tabular}

Although the similar samples differ, the parameters describing the basic shape of the heat affected area do not differ a lot. That is why we can use the average values for generating the basic shape of the pulse pit and the transition ring. Our second aim is to get the information if there is any dependence between the number of the laser pulses burned into the material and the shape of the sample. We can have a look at the real values again. The computed parameters for the set of samples with 10, 30, 50, 70 and 90 laser pulses burned into steel are shown in Table III. The dependencies of the parameters on the number of burned laser pulses are plotted in Figure 16.

Table III: Basic Parameters of the Set of Samples [ $\mu \mathrm{m}]$

\begin{tabular}{|l|l|l|l|l|l|}
\hline & $a$ & $b$ & $\begin{array}{l}\text { pit } \\
\text { depth }\end{array}$ & $\begin{array}{l}\text { ring } \\
\text { radius }\end{array}$ & $\begin{array}{l}\text { ring } \\
\text { height }\end{array}$ \\
\hline 10 & 21.0 & 25.13 & 3.81 & 16.41 & 2.8 \\
\hline 30 & 23.75 & 24.0 & 3.45 & 19.25 & 2.42 \\
\hline 50 & 32.38 & 35.88 & 6.47 & 13.78 & 2.3 \\
\hline 70 & 37.25 & 42.0 & 9.27 & 11.38 & 3.21 \\
\hline 90 & 40.25 & 46.13 & 7.08 & 10.91 & 2.3 \\
\hline
\end{tabular}

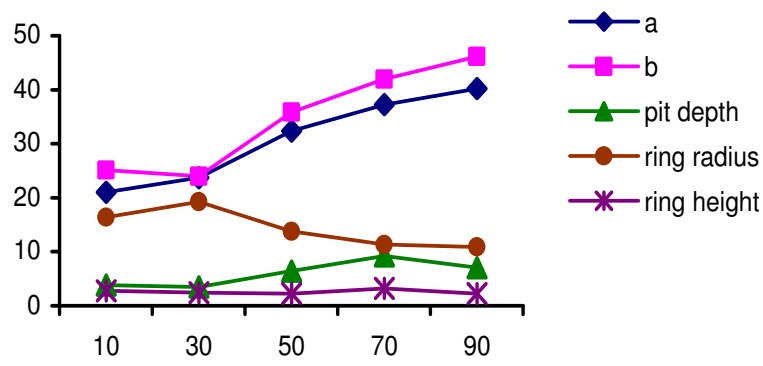

Figure 16: Parameters Dependence Plot

\section{Pulse Approximation}

The approximation was tested on various samples. The results for the area of the pit are very successful. More problematic is the area of the ring, where the roughness of the sample surface is high.

Results of approximation of the sample with 100 laser pulses burned into steel can be seen in Figure 17. In Figure 17a-c), cross-sections in the horizontal direction are shown, in Figure 17d-f), vertical cross-sections can be seen. The first cross-section from each triplet represents the middle of the pulse in the given direction, the others move more to the margin.

a)

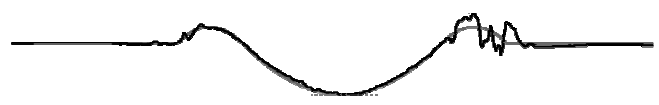

b)

c)

d)

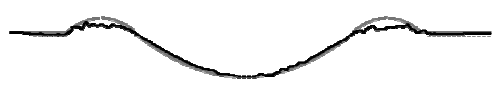

e)

f)
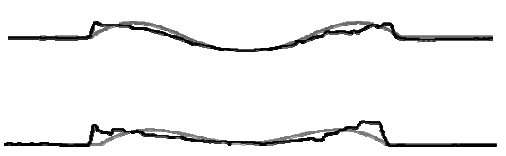

Figure 17: Approximation of 100 Laser Pulses Burned into Steel - Cross-Section Lines 
To compare the results in $3 \mathrm{D}$ view, see Figure 18. In the left column, the original samples are placed, the surface generated according to the parametric description is shown in the right column. For the comparison, samples with 10, 50, and 100 pulses burned into steel were chosen. All samples are watched from the same distance and direction.
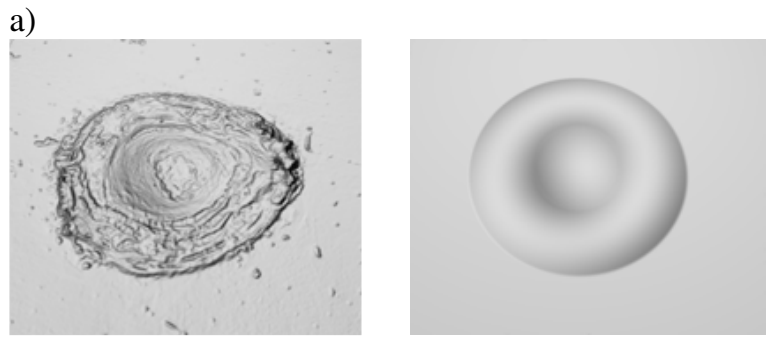

b)
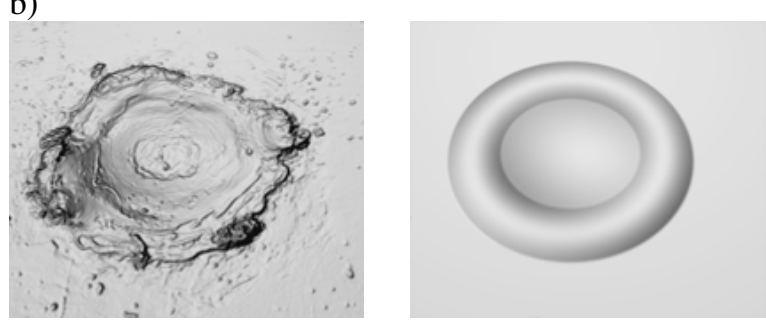

c)
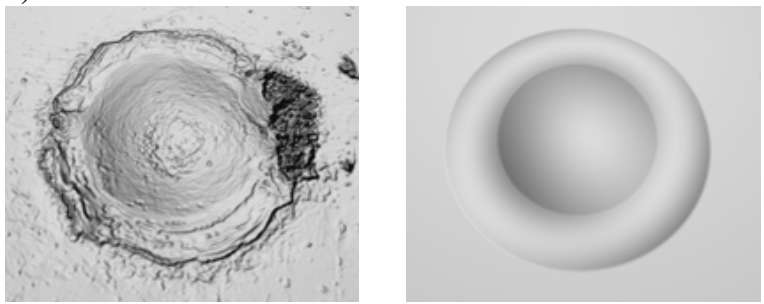

Figure 18: 3D Views at the Original and Generated Samples of 10, 50 and 100 Pulses Burned into Steel

\section{FUTURE PLANS, CONCLUSION}

The method described in this paper can be used for approximation of samples by a simplified, mathematically described smooth surface. To get more realistic results, we have to enhance the sample generation with random features such as methods for generating noise or various defects.

We are already experimenting with some of them. Present experiments show that the use of Perlin noise generating function (Perlin 1985), (Perlin 2002) can be the solution. The current results can be seen in Figure 19. In Figure 19a-b, a part of the transition ring of two different samples with the local roughness is shown, in Figure 19c, the surface generated by the Perlin noise function is depicted.

We are also testing number of further methods, e.g. modulation of concentric waves (irregular waves in a real sample can be seen in Figure 5c.), distortion, etc.
Of course for all these methods we need various parameters which would define roughness of the surface or the frequency of the local defects. Our task is also to design methods for an automatic parameterization of samples. a)

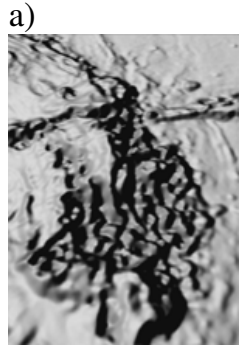

b)

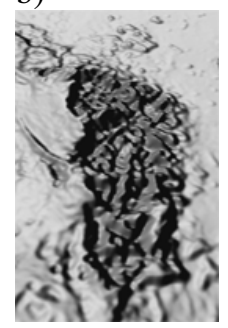

c)

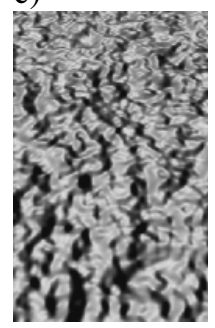

Figure 19: a-b) Local Defects of Two Different Samples, c) Surface Generated by the Perlin Noise Function

\section{ACKNOWLEDGEMENTS}

This work was supported by the Ministry of Education, Youth and Sport of the Czech Republic "University specific research -1311 ".

\section{REFERENCES}

Dahotre, N. B., Harimkar, S. P. 2008. Laser Fabrication and Machining of Materials, Springer, New York, USA.

Hájková, J. 2008. "Methods of Pulse Detection in Laser Simulation", In Proceedings of the 3rd International Conference on Software and Data Technologies ICSOFT 2008, ISBN: 978-989-8111-57-9, Porto, 186-191.

Perlin, K. 1985. "An Image Synthetizer", In Proceedings of the 12th annual conference on Computer graphics and interactive techniques, ISBN: 0-89791-166-0. ACM New York, 287-296.

Perlin, K. 2002. "Improving noise", In Proceedings of the 29th annual conference on Computer graphics and interactive techniques, ISBN: 0730-0301. ACM New York, 681-682.

Rektorys, K. 1981. Přehled užité matematiky. SNTL Praha.

Weisstein, E. W. 1999. The CRC Concise Encyclopedia of Mathematics. ISBN: 0-8493-9640-9, CRC Press LLC, USA.

\section{AUTHOR BIOGRAPHIES}

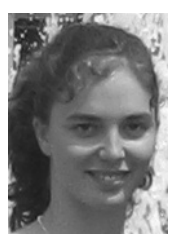

JANA HÁJKOVÁ is a PhD candidate and teaching assistant of computer science at the University of West Bohemia. Her research interests include data visualization and computer simulation. She received a MS in computer science from the University of West Bohemia in 2005. Contact her at hajkova@kiv.zcu.cz 